\title{
ARMSTRONG ON PROBABILISTIC LAWS OF NATURE
}

\author{
Jonathan D. Jacobs and Robert J. Hartman ${ }^{1}$
}

\begin{abstract}
D. M. Armstrong famously claims that deterministic laws of nature are contingent relations between universals and that his account can also be straightforwardly extended to irreducibly probabilistic laws of nature. For the most part, philosophers have neglected to scrutinize Armstrong's account of probabilistic laws. This is surprising precisely because his own claims about probabilistic laws make it unclear just what he takes them to be. We offer three interpretations of what Armstrong-style probabilistic laws are, and argue that all three interpretations are incompatible either with some feature of Armstrong's broader metaphysics or with essential features of his account of laws (or both).
\end{abstract}

D. M. Armstrong (among others) famously claims that deterministic laws of nature are contingent relations between universals and that his account can also be straightforwardly extended to irreducibly probabilistic laws of nature. ${ }^{2}$ For the most part, philosophers have neglected to scrutinize Armstrong's account of probabilistic laws. ${ }^{3}$ This is surprising precisely because his own claims about probabilistic laws make it unclear just what he takes them to be. We offer three interpretations of what Armstrong-style probabilistic laws are, and argue that all three interpretations are incompatible either with some feature of Armstrong's broader metaphysics or with essential features of his account of laws (or both).

\footnotetext{
${ }^{1}$ Order of authorship was determined by a coin flip.

${ }^{2}$ Fred Dretske (1977) and Michael Tooley (1977) also hold the view that laws of nature are relations between universals.

${ }^{3}$ As Duncan Maclean (2012, p. 1) states, there are only two philosophers besides himself who have takenup the topic of Armstrong's account of probabilistic laws. Bas van Fraassen $(1987 ; 1989)$ argues from statistical laws in philosophy of science to the claim that Armstrong's account of probabilistic laws are not able to generate the correct probabilities. Duncan Maclean (2012) continues the debate by arguing that Armstrong's (1988; 1997, pp. 240-241) reply to van Fraassen does not adequately resolve the problem. Our project differs because it concerns the metaphysics of laws themselves and not objective probabilities in the philosophy of science. And Gürol Irzik (1991) argues that Armstrong (1983, pp. 128-131) fails to deal adequately with the problem of how probabilistic laws are related to states of affairs when the law fails to be instantiated in felicitous circumstances. While our argument differs because it concerns the metaphysics of laws themselves, we enlist part of Irzik's argument in our broader argument against the third interpretation of Armstrong-style probabilistic laws.
} 


\section{Armstrong on Laws}

Armstrong's $(1983 ; 1997)$ view of laws is embedded within his wider metaphysical commitments about states of affairs, universals, and causation. A state-of-affairs is a particular having a monadic property, such as the chalk's being white, or is at least two particulars standing in some relation, such as the chair's being four feet to my left. ${ }^{4}$ The property or relation that partially composes a state-of-affairs is an immanent or Aristotelian universal - the kind of universal that is dependent on the concrete world in a particular way. Universals are abstractions from states of affairs and so are state-of-affairs types (Armstrong 1997, p. 38). This claim is often called the principle of abstraction. Because universals are abstractions, they must be instantiated in some state-of-affairs at some time in order to exist (Armstrong 1997, p. 227). This claim is often called the principle of instantiation.

According to Armstrong (1997, pp. 226-227), a law of nature is a 'structural' universal that is itself 'a certain linking of universals.' The kinds of universals included in the structure of a law include a dyadic necessitation relation and the two universals that it relates. Because laws of nature are themselves universals, laws must be instantiated in some state-of-affairs or other in order to exist. In other words, laws are abstractions from states of affairs and so are state-ofaffairs types. More specifically, a law is an abstraction from a complex state-of-affairs in which one state-of-affairs necessitates another. Consider the complex state-of-affairs Ben's smoking causes Ben's having cancer. In that case, b's being S (Ben's smoking) necessitates b's being C

\footnotetext{
${ }^{4}$ Since particulars that stand in some relation can be states of affairs, there can be states of affairs involving other states of affairs, as it happens with laws of nature. Thanks to an anonymous referee from this journal for emphasizing this point.
} 
(Ben's having cancer). In Armstrong's shorthand, the complex state-of-affairs is N(Sb,Cb), and the law abstracted from it is $\mathrm{N}(\mathrm{S}, \mathrm{C})$.

Laws are responsible for pushing and pulling in the world. Armstrong (1997, pp. 218219) subsumes all singular causation, one particular state-of-affairs causing another, under the laws of nature. Thus, each instance of causation is an instantiation of a law of nature. Laws of nature may be either deterministic or irreducibly probabilistic. In the case of an irreducibly probabilistic law, represented as, say, $\mathrm{N}_{0.8}(\mathrm{~F}, \mathrm{G})$, there is a 0.8 objective probability that when an F-type state-of-affairs such as Fa obtains, that Fa necessitates $\mathrm{Ga}^{5}$ According to Armstrong (1983, p. 132; 1997, p. 237), deterministic laws are the limiting case of irreducibly probabilistic laws. In the case of a deterministic law, say, $\mathrm{N}_{1.0}(\mathrm{~F}, \mathrm{G})$, there is an objective probability of 1.0 that when some F-type state-of-affairs such as Fa occurs, that Fa necessitates Ga.

\section{Three Interpretations}

Armstrong makes several further claims that make it unclear what state-of-affairs obtains upon the instantiation of a probabilistic law. But since a probabilistic law is an abstraction from a state-of-affairs, the metaphysics of laws themselves is unclear. To clarify Armstrong's view, we must answer two questions: (i) what is the form of a probabilistic law and (ii) what is the form of its instantiation? In what follows, we consider some of Armstrong's claims about probabilistic laws and how, taking the claims as interpretive fixed points, we might answer these two questions.

${ }^{5}$ Armstrong (1997, p. 230) holds that some deterministic and probabilistic laws may be 'iron' and others 'defeasible.' Iron laws hold without qualification, and defeasible laws hold provided nothing else interferes. We ignore this complication. 
First, Armstrong states that ordinary causation occurs when either a deterministic or probabilistic law is instantiated.

Necessitation is just the same old relation found in any actual case of a (token) cause bringing about a (token) effect, whether governed by a deterministic law, probabilistic law or no law at all (1988, p. 226, italics ours).

[O]rdinary causation occurs whether the law governing the causation is deterministic or merely probabilistic (1997, p. 238, italics ours).

Because Armstrong construes the instantiation of deterministic and probabilistic laws as 'the same old relation' and 'ordinary causation,' one might be tempted to hold the view that there is no metaphysical difference between the instantiation of a deterministic law and a probabilistic law. On this view, laws of nature are dyadic relations between universals $-\mathrm{N}_{0.8}(\mathrm{~F}, \mathrm{G})$ in the indeterministic case, $\mathrm{N}_{1.0}(\mathrm{~F}, \mathrm{G})$, in the deterministic case - and their instantiations are, in both cases, the very same state-of-affairs: $\mathrm{N}(\mathrm{Fa}, \mathrm{Ga})$. Whether it is a deterministic or probabilistic law that smoking nomically ensures cancer, any particular instance of smoking ensuring cancer is just the necessitation relation. Call the view, on which the instantiations of deterministic laws have the same form as the instantiations of probabilistic laws, the identity interpretation.

Second, Armstrong routinely asserts that probabilistic laws involve a probability of causation-and not probabilistic causation.

I hold that a probabilistic law gives the probability of a necessitation in a particular case (1988, p. 226).

What a probabilistic causal law gives us is not probabilistic causality but a certain probability that causation will occur (1997, p. 238).

These passages suggest a slightly different interpretation of probabilistic laws that highlights the probability of necessitation. The central idea is that a probabilistic law is a monadic property of a 
relational state-of-affairs type. That is, the instantiation of a probabilistic law is identical with a probabilistic fact about one state-of-affairs necessitating another. It is not that smoking probabilifies cancer, but rather that there is a certain probability that a certain state-of-affairs, smoking necessitating cancer, will be instantiated. On this view, the form of a probabilistic law includes four universals and may be symbolized as $\mathrm{P}_{0.8}(\mathrm{~N}(\mathrm{~F}, \mathrm{G}))$, which means that there is a 0.8 objective probability of necessitation occurring between F-type and G-type states of affairs. Since deterministic laws are the limiting case of probabilistic laws, a deterministic law is properly symbolized as $\mathrm{P}_{1.0}(\mathrm{~N}(\mathrm{~F}, \mathrm{G}))$. Their respective instantiations are the type-distinct states of affairs $\mathrm{P}_{0.8}(\mathrm{~N}(\mathrm{Fa}, \mathrm{Ga}))$ and $\mathrm{P}_{1.0}(\mathrm{~N}(\mathrm{Fa}, \mathrm{Ga}))$. The difference between such instantiations lies in their monadic properties, $\mathrm{P}_{0.8}$ and $\mathrm{P}_{1.0}$. Call this the monadic interpretation. An important feature of this interpretation is that the same necessitation relation obtains whether a deterministic or probabilistic law is instantiated, and so it nicely incorporates Armstrong's (1988, p. 226) claim that deterministic and probabilistic laws involve 'the same old relation.'

Third, one might instead interpret Armstrong as asserting that there are different types of necessitation universals - some that are deterministic and others that are probabilistic.

If the relation between $\mathrm{F}$ and $\mathrm{G}$ is $(\mathrm{N}: 1)$ then there logically must be necessitation in each singular case. If the relation is $(\mathrm{N}: \mathrm{P})$ where $\mathrm{P}$ is less than 1 , then we have a certain objective probability of necessitation in each singular case (1983, p. 132).

One might construe Armstrong's claim about $(\mathrm{N}: 1)$ and $(\mathrm{N}: \mathrm{P})$ as a claim that these universals are distinct causal relations in the same general class. On this interpretation, laws of nature are dyadic relations between universals, such as $\mathrm{N}_{0.8}(\mathrm{~F}, \mathrm{G})$ and $\mathrm{N}_{1.0}(\mathrm{~F}, \mathrm{G})$, and their respective instantiations are the following distinct kinds of state-of-affairs: $\mathrm{N}_{0.8}(\mathrm{Fa}, \mathrm{Ga})$ and $\mathrm{N}_{1.0}(\mathrm{Fa}, \mathrm{Ga})$. On this view, there are a plurality of distinct $\mathrm{N}$ relations such as $\mathrm{N}_{0.71}, \mathrm{~N}_{0.8}$, and $\mathrm{N}_{1.0}$, and the 
instantiation of each distinct nomic relation is a distinct kind causal relation. Thus, no causal relation is common to all instantiations of deterministic and probabilistic laws. But since these distinct causal relations are in the same class, there is a sense in which all instantiations of a law include the same old relation. Call this the plurality interpretation.

It is our contention that the interpretations summarized below exhaust all the plausible metaphysical characterizations of Armstrong's view of probabilistic laws.

\begin{tabular}{|l|c|c|c|}
\hline & Identity Interpretation & Monadic Interpretation & Plurality Interpretation \\
\hline $\begin{array}{l}\text { Deterministic } \\
\text { Law } \\
\text { Law }\end{array}$ & $\mathrm{N}_{1.0}(\mathrm{~F}, \mathrm{G})$ & $\mathrm{P}_{1.0}(\mathrm{~N}(\mathrm{~F}, \mathrm{G}))$ & $\mathrm{N}_{1.0}(\mathrm{~F}, \mathrm{G})$ \\
$\begin{array}{l}\text { Deterministic } \\
\text { Instantiation }\end{array}$ \\
$\begin{array}{l}\text { Probabilistic } \\
\text { Instantiation }\end{array}$
\end{tabular}

We now turn to showing the way in which each interpretation conflicts with some feature of Armstrong's broader metaphysics or account of laws (or both).

\section{Against the Identity Interpretation}

A central feature of the identity interpretation is that the same concrete state-of-affairs, $\mathrm{N}(\mathrm{Fa}, \mathrm{Ga})$, is the instantiation of both the deterministic law $\mathrm{N}_{1.0}(\mathrm{~F}, \mathrm{G})$ and the probabilistic law $\mathrm{N}_{0.8}(\mathrm{~F}, \mathrm{G})$. The problem with this interpretation is simple, and can best be seen by asking this question: what law is abstracted from $\mathrm{N}(\mathrm{Fa}, \mathrm{Ga})$ ? Because laws are abstractions from concrete states of affairs, the answer is plausibly $\mathrm{N}(\mathrm{F}, \mathrm{G})$. And if $\mathrm{N}(\mathrm{F}, \mathrm{G})$ were the same as $\mathrm{N}_{1.0}(\mathrm{~F}, \mathrm{G})$, then it must also be 
the same as $\mathrm{N}_{0.8}(\mathrm{~F}, \mathrm{G})$, but this cannot be the case since by hypothesis $\mathrm{N}_{0.8}$ is not $\mathrm{N}_{1.0}$. Because both $\mathrm{N}_{0.8}(\mathrm{~F}, \mathrm{G})$ and $\mathrm{N}_{1.0}(\mathrm{~F}, \mathrm{G})$ cannot be abstracted from $\mathrm{N}(\mathrm{Fa}, \mathrm{Ga})$, it follows that at most one of the two laws exists! Call this difficulty the abstraction problem. The crux of the abstraction problem is that the identity interpretation implies that a state-of-affairs token can be typeidentical with distinct state-of-affairs types. And this implication is squarely incompatible with Armstrong's commitment that a state-of-affairs token can have only one state-of-affairs type. After all, types and tokens are the very same universals on Armstrong's view.

One might think that there is a way to understand Armstrong's account of laws on the identity interpretation that escapes the abstraction problem. On this view, a deterministic and probabilistic law can be abstracted from the same state-of-affairs token $\mathrm{N}(\mathrm{Fa}, \mathrm{Ga})$ under different extrinsic conditions. What are the relevant conditions? A deterministic law is abstracted from $\mathrm{N}(\mathrm{Fa}, \mathrm{Ga})$ in the case that all actual F-type states of affairs necessitate G-type states of affairs, and a probabilistic law is abstracted from $\mathrm{N}(\mathrm{Fa}, \mathrm{Ga})$ in the case that some but not all actual F-type states of affairs necessitate G-type states of affairs. The extrinsic fact that determines whether the abstraction of $\mathrm{N}(\mathrm{Fa}, \mathrm{Ga})$ is a deterministic or probabilistic law is the actual distribution of F-type states of affairs that necessitate G-type states of affairs. In other words, the pattern of actual Ftype states of affairs that necessitate G-type states of affairs is the truth-maker for whether the abstraction of $\mathrm{N}(\mathrm{Fa}, \mathrm{Ga})$ is a deterministic or probabilistic law. Call this alternative version of the identity interpretation the extrinsic identity interpretation.

There are several problems with the extrinsic identity interpretation. One problem is that the extrinsic identity interpretation substantively revises Armstrong's account of laws. Armstrong (1983, p. 77; 1997, p. 223) construes a law of nature as a mere relational state-of- 
affairs type. On the extrinsic identity interpretation, however, a law is identical with a relational state-of-affairs type and the frequency of its occurrence.

Another difficulty of this nomic revision is that the extrinsic identity interpretation at least partially inverts the order of explanation between laws and regularities. For Armstrong (1997, p. 223, p. 235; 1983, pp. 103-104), laws of nature are responsible for all the pushing and pulling in the world. For this reason, laws fully explain regularities. In particular, the deterministic law $\mathrm{N}(\mathrm{F}, \mathrm{G})$ fully explains why there is the regularity that 'all Fs are Gs': the law is instantiated anytime a solitary F-type state-of-affairs obtains. On the extrinsic identity interpretation, however, regularities are partial truth-makers for laws and so constitute a partial ground of explanation for the nature of the law. But then, the law $N(F, G)$ does not fully explain the regularity 'all Fs are Gs', because the regularity 'all Fs are Gs' is a partial metaphysical explanation of the law.

A related problem for the extrinsic identity interpretation is that it fails to support counterfactuals. For Armstrong (1997, p. 223, p. 245), laws such as N(F,G) are 'strong' in a way that support counterfactuals about what would occur if some non-actual state-of-affairs such as Fa were to obtain. But take the abstraction $\mathrm{N}(\mathrm{F}, \mathrm{G})$ from the state of affairs $\mathrm{N}(\mathrm{Fa}, \mathrm{Ga})$. The abstraction is a deterministic law in virtue of the fact that all actual F-type states of affairs necessitate G-type states of affairs. The law N(F,G), however, does not guarantee that some nonactual F-type state of affairs Fa would necessitate $\mathrm{Ga}$, because the nature of the law would be partially determined by whether Fa would necessitate Ga. In the case in which Fa would not necessitate $\mathrm{Ga}, \mathrm{N}(\mathrm{F}, \mathrm{G})$ would not be a deterministic law but rather the probabilistic law $\mathrm{N}_{0.99}(\mathrm{~F}, \mathrm{G})$. And in the case in which Fa would necessitate $\mathrm{Ga}$, the deterministic law $\mathrm{N}(\mathrm{F}, \mathrm{G})$ 
would hold. But the law holds in virtue of the fact that Fa necessitates Ga, and thus the complex state-of-affairs $\mathrm{N}(\mathrm{Fa}, \mathrm{Ga})$ does not obtain strictly in virtue of the law.

A further problematic result of this nomic revision is that the extrinsic identity interpretation conflates the law's probability of instantiation with the frequency of its being instantiated. For Armstrong (1997, pp. 237-239), the law $\mathrm{N}_{0.8}(\mathrm{~F}, \mathrm{G})$ has a 0.8 'single-case chance' of being instantiated for each F-type state-of-affairs. As a result, the propensity that $\mathrm{N}_{0.8}(\mathrm{~F}, \mathrm{G})$ bestows on particular F-type states of affairs may diverge without contradiction from the actual frequency of F-type states of affairs that necessitate G-type states of affairs. To be more concrete, the law $\mathrm{N}_{0.8}(\mathrm{~F}, \mathrm{G})$ is compatible with every actual F-type state-of-affairs necessitating a G-type state-of-affairs and with a very small proportion of actual F-type states of affairs necessitating G-type states of affairs. On the extrinsic identity interpretation, however, the law $\mathrm{N}_{0.8}(\mathrm{~F}, \mathrm{G})$ is incompatible with the frequency in which every F-type state-of-affairs necessitates a G-type state-of-affairs, because a probabilistic law can be abstracted from N(Fa,Ga) only when some but not all F-type states of affairs necessitate G-type states of affairs.

\section{Against the Monadic Interpretation}

The monadic interpretation is that a law of nature is identical with a certain kind of monadic property of a certain kind of relational state-of-affairs type. On this interpretation, a probabilistic law is $\mathrm{P}_{0.8}(\mathrm{~N}(\mathrm{~F}, \mathrm{G}))$, and a deterministic law is $\mathrm{P}_{1.0}(\mathrm{~N}(\mathrm{~F}, \mathrm{G}))$. The monadic interpretation does not suffer from the abstraction problem, because the instantiations of these laws, $\mathrm{P}_{0.8}(\mathrm{~N}(\mathrm{Fa}, \mathrm{Ga}))$ and $\mathrm{P}_{1.0}(\mathrm{~N}(\mathrm{Fa}, \mathrm{Ga}))$, are not identical in virtue of the fact that their monadic properties differ.

One implication of the monadic interpretation is that it slightly revises Armstrong's account of laws by adding a fourth universal to the structure of a law. Armstrong (1983, p. 77; 
1997, p. 223) construes a law of nature as a mere relational state-of-affairs type. On the monadic interpretation, however, a probabilistic law is identical with a monadic property of a relational state-of-affairs type. The same is true of a deterministic law, because deterministic laws are the limiting case of probabilistic laws.

Another implication of this interpretation is that it substantively revises Armstrong's account of causation. According to Armstrong, causation is identical with the necessitation relation between states of affairs (1997, pp. 228-229) and the instantiation of a law (1997, p. 219). The monadic interpretation, however, implies that causation is identical with the necessitation relation between states of affairs but not the instantiation of a law. For the instantiation of a law is not identical with one state-of-affairs necessitating another-but rather with a probabilistic fact about one state-of-affairs necessitating another.

An even more pressing difficulty is that probabilistic laws may fail to be strong on the monadic interpretation. A law is 'strong,' according to Armstrong (1997, p. 223), if it is responsible for pushing and pulling in the world. Strong laws explain regularities such as 'all Fs are Gs' that occur in the world (1997, p. 235), support counterfactuals about what would occur if some non-actual state-of-affairs Fa were to obtain (1997, p. 245), and legitimate rational inductive inferences about the shape of future regularities (1983, pp. 103-104). ${ }^{6}$ The monadic interpretation faces a problem if laws do not turn out to be strong, because Armstrong (1983, pp. 39-74; 1997, p. 235) argues that regularity and best-system Humean accounts of laws are implausible precisely because they are not strong.

\footnotetext{
${ }^{6}$ This picture is somewhat complicated by the fact that while Armstrong (1983, p. 79) did not initially allow laws of nature to change within a particular possible world, he (1997, pp. 257-258) has been convinced that he should allow it. As a result, laws may not always explain the same regularities, support the same counterfactuals, or legitimate the same inductive inferences. Nevertheless, in the spatiotemporal region where a particular law of nature is operative - and it may be all of spacetime if the law doesn't change - the law explains regularities, supports counterfactuals, and legitimates inductive inferences.
} 
But why think that probabilistic laws may not be strong on this interpretation? The law $\mathrm{P}_{0.8}(\mathrm{~N}(\mathrm{~F}, \mathrm{G}))$ plausibly indicates that when and only when both F- and G-type states of affairs obtain, there is a 0.8 objective probability that the relation $\mathrm{N}$ holds between them. That is, there is a 0.8 objective probability that a F-type state-of-affairs such as Fa necessitates a G-type stateof-affairs such as Ga in the case and only in the case in which Fa and Ga both obtain. But then, the law $\mathrm{P}_{0.8}(\mathrm{~N}(\mathrm{~F}, \mathrm{G}))$ does not transparently imply that any solitary F-type state-of-affairs such as Fa has a 0.8 objective probability of necessitating a G-type state-of-affairs. After all, the 0.8 objective probability of $\mathrm{N}$ relating F- and G-type states of affairs holds only for the case in which both F- and G-type states of affairs obtain. For the same reason, the law $\mathrm{P}_{0.8}(\mathrm{~N}(\mathrm{~F}, \mathrm{G}))$ does not obviously imply that if a solitary state-of-affairs Fa were to obtain, then there is a 0.8 objective probability that Fa would necessitate Ga. But since these implications are required by strong laws and are supposed to fall out of the metaphysics of laws, probabilistic laws are not obviously strong on the monadic interpretation. ${ }^{7}$

\section{Against the Plurality Interpretation}

The central feature of the plurality interpretation is that different laws have different kinds of nomic relations. On this interpretation, a probabilistic law has the form $\mathrm{N}_{0.8}(\mathrm{~F}, \mathrm{G})$ and a deterministic law has the form $\mathrm{N}_{1.0}(\mathrm{~F}, \mathrm{G})$. The plurality interpretation is not subject to the abstraction problem, because the causal relations intrinsic to their instantiations, $\mathrm{N}_{0.8}(\mathrm{Fa}, \mathrm{Ga})$ and $\mathrm{N}_{1.0}(\mathrm{Fa}, \mathrm{Ga})$, are not the same. The plurality interpretation also retains Armstrong's account of laws as mere dyadic relations between universals and account of causation as the instantiation of a law, which is an improvement over the monadic interpretation.

\footnotetext{
${ }^{7}$ John Bolender (2006) argues that Armstrong's general account of laws is not strong for a different reason. Roughly, his argument is that since laws 'logically supervene' on states of affairs, they cannot account for robust causal links between states of affairs.
} 
An initial problem with the plurality interpretation is that it reduces the parsimony of Armstrong's metaphysics. In particular, it greatly increases the number of different causal relations in his metaphysics. How many causal relations are there? The number of distinct causal relations is the same as the number of laws with different objective probabilities of being instantiated in felicitous conditions. The plurality interpretation, then, is less parsimonious than the identity and monadic interpretations at least with regard to causation, because the latter interpretations employ only one kind of causal relation.

A more substantial difficulty for the plurality interpretation is that the instantiation of a probabilistic law is most plausibly construed as probabilistic causation. Why think that the instantiation of a probabilistic law on this interpretation is an instance of probabilistic causation? Probabilistic causation is the kind of causal relation that may obtain with or without its second term. And the most plausible way to read off the metaphysics of the state-of-affairs $\mathrm{N}_{0.8}(\mathrm{Fa}, \mathrm{Ga})$ is to read it as a successful case of probabilistic causation. After all, there is no monadic property of the relational state-of-affairs type indicating a probability of causation. Furthermore, as Gürol Irzik (1991, p. 215) plausibly argues, probabilistic laws cover or govern relevant states of affairs whether or not causation occurs, because all states of affairs are covered by the laws of nature. Armstrong (1983, p. 135) simply denies that laws govern all states of affairs. ${ }^{8}$ But Armstrong's denial is counterintuitive, because it is plausible to think that there are no states of affairs that the laws of nature do not cover. So then, on the supposition that the law $\mathrm{N}_{0.8}(\mathrm{~F}, \mathrm{G})$ covers $\mathrm{Fa}$ whether or not causation occurs and that causation fails to occur, the form of the resultant state-of-affairs

8 ' $[\mathrm{C}]$ onsider $a$, an F, which is not G. Object $a$ has a certain probability, $\mathrm{P}$, of being $\mathrm{G}$, but the probability is not realized. Is not this probability a pure unrealized potentiality of $a$ to be G? I trust that this is not so. I want to say that $a$ is $\mathrm{F}$, that it has various other categorical properties, and that is the end of the matter as far as $a$ is concerned. It is certainly true that $a$ had a certain probability of being G. But the truth-maker for this truth is to be found in the positive instantiations of the probabilistic law' (Armstrong 1983, p. 135). 
is plausibly $\mathrm{N}_{0.8}(\mathrm{Fa}, \mathrm{G}) .{ }^{9}$ And that particular state-of-affairs is an instance of failed probabilistic causality.

Armstrong (1997, p. 238) repudiates probabilistic causation for its obvious problems. A conceptual problem is that an instance of failed probabilistic causation is a causal relation between something and nothing. A metaphysical problem is that an instance of failed probabilistic causation is ruled out by Armstrong's metaphysical commitments. After all, the form of a probabilistic causation failure is $\mathrm{N}_{0.8}(\mathrm{Fa}, \mathrm{G})$, and this state-of-affairs violates the principle of instantiation. The universal $\mathrm{G}$ is a state-of-affairs type without its token.

One might be tempted to think that a failed instance of probabilistic causation is not incompatible with Armstrong's metaphysics, because one might think that the state-of-affairs $\mathrm{N}_{0.8}(\mathrm{Fa}, \mathrm{G})$ is quasi-universal. For Armstrong (1997, p. 256), a 'quasi-universal' is a particularized universal of the following form: $\mathrm{N}(\mathrm{Fa}, \mathrm{G})$. To illustrate this concept, consider an example from Michael Tooley (1977, pp. 686-687):

All the fruit in Smith's garden at any time are apples. When one attempts to take an orange into the garden, it turns into an elephant. Bananas so treated become apples as they cross the boundary, while pears are resisted by a force that cannot be overcome. Cherry trees planted in the garden bear apples, or they bear nothing at all. If all these things were true, there would be a very strong case for its being a law that all the fruit in Smith's garden are apples.

In this example, there is a local law that governs only Smith's Garden. The law has the form $\mathrm{N}(\mathrm{Fa}, \mathrm{G})$. The particular Fa, fruit in Smith's garden, is the antecedent relatum of the law, and the

\footnotetext{
${ }^{9}$ An anonymous referee from another journal pointed out that a proposal in a related context from Markus Schrenk (2011, pp. 587-591) may be adapted to help Armstrong. Put simply, $\mathrm{N}_{0.8}$ is not a causal relation but is a force that tends toward the production of $\mathrm{G}$ but sometimes fails. So, the law $\mathrm{N}_{0.8}(\mathrm{Fa}, \mathrm{G})$ covers the state-of-affairs Fa even when causation fails to occur, because $\mathrm{N}_{0.8}$ is instantiated but is not causation. Schrenk (2011, p. 589) notes that Armstrong was open to this idea in private communication. The problem with this move is that Armstrong identifies the instantiated necessitation relation with causation, and denying that claim when it runs into trouble is ad hoc.
} 
universal G, being an apple, is its consequent relatum. Armstrong (1997, p. 256) calls this particularized universal a 'bastard state-of-affairs type,' because the universal $\mathrm{N}(\mathrm{Fa}, \mathrm{G})$ includes a particular in abstraction. The quasi-law $\mathrm{N}(\mathrm{Fa}, \mathrm{G})$, however, does not violate the principle of instantiation, because it takes the form $\mathrm{N}(\mathrm{Fa}, \mathrm{G})$ only in abstraction. When the law is instantiated, the state-of-affairs $\mathrm{N}(\mathrm{Fa}, \mathrm{Ga})$ obtains. But then, a failed instance of probabilistic causation cannot be a quasi-universal, because a failed instance of probabilistic causation is an instantiated causal relation without its effects such as $\mathrm{N}(\mathrm{Fa}, \mathrm{G})$. The upshot is that one cannot avoid the metaphysical problem with probabilistic causality by appealing to quasi-universals.

It is not the case, however, that a proponent of the plurality interpretation must endorse probabilistic causality with its aforementioned conceptual and metaphysical problems. One way to evaluate the options before the proponent of the plurality interpretation is to consider different ways to respond to an inconsistent triad. The first claim is an implication of the plurality interpretation, and the latter claims are supplied by Armstrong's own commitments.

(1) The instantiation of a probabilistic law is probabilistic causation.

(2) Causation is not probabilistic.

(3) Causation is the instantiation of a probabilistic law.

So, how might one affirm the plurality interpretation—and so affirm (1) but escape the inconsistent triad? One might want to deny (2) —and so affirm probabilistic causation. The resultant view is that the instantiation of a probabilistic law is probabilistic causation, and it is subject to the aforesaid conceptual and metaphysical difficulties.

Alternatively, one might want to deny (3) — and so separate Armstrong's account of probabilistic laws from his account of causation. But there are also difficulties here. On the one hand, one might want to retain the commitment that causation is the instantiation of a 
deterministic law but separate causation from the instantiation of a probabilistic law. In this case, there is an asymmetry between his account of deterministic and probabilistic laws. One revisionary implication of this view is that deterministic laws cannot be the limiting case of probabilistic laws, because they are no longer relations in the same class. Another revisionary implication is that probabilistic laws are not strong, because their instantiations are not identical with singular causal relations. On the other hand, one might want separate causation from the instantiation of both deterministic and probabilistic laws. Certainly, this supposition avoids the problem that deterministic and probabilistic laws are relations in different classes. It avoids that problem, however, only by compounding the second difficulty so that neither probabilistic nor deterministic laws are strong. This result undermines the feature of Armstrong's view that makes it preferable to regularity or best-systems accounts of laws of nature.

\section{Concluding Thoughts}

Armstrong's metaphysics of probabilistic laws is between a rock and a hard place, because there is no plausible interpretation of it that coheres with his wider metaphysical commitments. The central problem with the identity interpretation is that it is incompatible with Aristotelian universals, and the extrinsic version of the identity interpretation is incompatible with several of Armstrong's central claims about laws. The monadic interpretation slightly revises the metaphysics of laws, substantively revises the metaphysics of causation, and makes it unclear whether deterministic or probabilistic laws are strong. The plurality interpretation reduces the parsimony of Armstrong's metaphysics, and, since the instantiation of a probabilistic law amounts to probabilistic causation, this interpretation must either accept the problems with probabilistic causation or separate causation from the instantiation of (at least) probabilistic laws. 
Therefore, each interpretation either fails to capture essential features of Armstrong's of probabilistic laws or is incompatible with some feature of his metaphysics (or both).

Is there an interpretation of Armstrong-style probabilistic laws that is least problematic? If there is a least problematic option, it is not obvious. Which problems one takes to be least unattractive will depend greatly on one's broader systematic metaphysical commitments. We conclude that Armstrong's metaphysics of probabilistic laws is problematic and none of the interpretations on offer are obviously preferable to the others. ${ }^{10}$

${ }^{10}$ We are grateful to Errin Clarke, Jeremy Skrzypek, John Heil, and two anonymous referees from this journal for comments on this paper. 


\section{REFERENCES}

Armstrong, D. M. (1983) What is a Law of Nature? (Cambridge: Cambridge University Press).

Armstrong, D. M. (1988) 'Reply to van Fraassen,' Australasian Journal of Philosophy 66, 224229.

Armstrong, D. M. (1997) A World of States of Affairs (Cambridge: Cambridge University Press).

Bolender, John (2006) 'Nomic Universals and Particular Causal Relations: Which are Basic and Which are Derived?’ Philosophia 34, 405-410.

Dretske, Fred I. (1977) 'Laws of Nature,' Philosophy of Science 44, 248-268.

Irzik, Gürol (1991) 'Armstrong's Account of Probabilistic Laws,' Analysis 51, 214-217.

Maclean, Duncan (2012) 'Armstrong and van Fraassen on Probabilistic Laws of Nature,' Canadian Journal of Philosophy 42, 1-14.

Schrenk, Markus (2011) 'Inferring with Nomological Necessity,' The Philosophical Quarterly 61, 577-597.

Tooley, Michael (1977) 'Laws of Nature,' Canadian Journal of Philosophy 7, 667-698.

van Fraassen, Bas C. (1987) 'Armstrong on Laws and Probabilities,' Australasian Journal of Philosophy 65, 243-260.

van Fraassen, Bas C. (1989) Laws and Symmetries (Oxford: Oxford University Press). 